\title{
Systematic review of the accuracy of antibody tests used to screen asymptomatic adults for hepatitis $\mathrm{C}$ infection
}

\author{
Geneviève Cadieux PhD MD, Jennifer Campbell MD MPH, Nandini Dendukuri PhD
}

Abstract

Background: Several expert groups, including the United States Preventive Services Task Force and the Canadian Task Force on Preventive Health Care, have recently examined or are currently examining whether primary care physicians should screen asymptomatic adults for hepatitis $\mathrm{C}$ virus (HCV) infection. To inform decision-making on HCV screening, we performed a systematic review of the accuracy of antibody tests compared with other immunoassays and RNA detection for screening asymptomatic adults for HCV infection in Canada.

Methods: MEDLINE and Embase databases were searched from 1990 to 2016; resulting citations were uploaded into DistillerSR and independently screened by 2 reviewers. Original research studies, systematic reviews and meta-analyses were eligible for inclusion. At least $80 \%$ of the study population had to be asymptomatic, nonpregnant, treatment-naïve adults with unknown liver enzyme values and unknown HCV status. Risk of bias was assessed with the use of the Quality Assessment of Diagnostic Accuracy Studies version 2 (QUADAS-2) tool; the quality of the body of evidence was assessed by means of GRADE (Grading of Recommendations Assessment, Development and Evaluation) methodology.

Results: Of 1537 articles identified, 81 underwent full-text review, and 9 studies met the inclusion criteria. Compared with RNA detection, the sensitivity of the third-generation enzyme-linked immunosorbent assay was variable $(61.0 \%-81.8 \%)$, and its specificity was high (97.5\%-99.7\%). As expected, there were more false-positive results when comparing antibody tests to RNA detection than to other immunoassays. Our GRADE assessment suggested that there was a high concern for risk of bias, particularly verification bias, and substantial inconsistency between studies in terms of their design.

Interpretation: More research is needed to better characterize the accuracy of antibody tests used to screen for HCV infection in the general population. Jurisdictions that recently adopted birth cohort screening for HCV infection are encouraged to evaluate and report on the accuracy of HCV screening tests and screening benefits and harms. PROSPERO registration: no. CRD42016039710.

T he incidence of hepatitis $\mathrm{C}$ virus (HCV) infection in Canada has declined in recent years. ${ }^{1,2}$ The population prevalence of chronic $\mathrm{HCV}$ infection in this country is estimated at $0.64 \%-0.71 \%,{ }^{2}$ about half that in the United States. ${ }^{3}$ An estimated $21 \%-44 \%$ of Canadians with chronic HCV infection are unaware of their infection..$^{1,2}$ In low-prevalence countries such as Canada and the United Kingdom, the approach to prevention and control of HCV infection has focused on case-finding, ${ }^{4,5}$ i.e., testing people with risk factors for the infection, such as intravenous drug users and refugees from endemic countries. The recent development of effective but costly treatment for chronic hepatitis $\mathrm{C}^{6}$ has led some to reevaluate the evidence for and against population screening for HCV infection. ${ }^{7}$ In 2013, the US Preventive Services Task Force revised its 2004 recommendation against screening asymptomatic adults for $\mathrm{HCV}$ infection; ${ }^{8}$ it now recommends one-time screening for all adults born between 1945 and 1965..$^{7}$ The Canadian Task
Force on Preventive Health Care is examining whether primary care physicians should screen asymptomatic adults for HCV infection. ${ }^{9}$

Guidance from the World Health Organization ${ }^{10,11}$ and the UK National Screening Committee ${ }^{12}$ on when screening should be performed emphasizes the fundamental importance of having a "safe, valid, and reliable" screening test. Screening for $\mathrm{HCV}$ infection typically relies on antibody testing. Because antibodies may persist ${ }^{13}$ after $\mathrm{HCV}$ infection is spon-

Competing interests: None declared.

This article has been peer reviewed.

Correspondence to: Geneviève Cadieux, genevieve.cadieux@mail. utoronto.ca

CMAJ Open 2016. DOI:10.9778/cmajo.20160084 
taneously cleared (which occurs in about $25 \%$ of those infected ${ }^{14}$ ), antibody testing cannot discriminate current from resolved infections, which leads to false-positive results. ${ }^{15}$ False-positive results can also occur when other antibodies interact nonspecifically with the test. ${ }^{16}$ False-positive results can cause harm (e.g., through labelling and anxiety). People with a positive screening result typically undergo further testing, which has resource implications and may carry additional inherent risk. To inform decision-making on screening for chronic HCV infection in Canada, we performed a systematic review of the evidence on the accuracy of antibody tests used to screen asymptomatic adults for HCV infection.

\section{Methods}

\section{Research question}

Our objective was to carry out a systematic review to estimate the accuracy of antibody tests used in Canada to screen for HCV infection among asymptomatic, nonpregnant, treatmentnaïve adults with unknown liver enzyme values. We also sought to assess the accuracy of the 2-step HCV screening procedure (i.e., the combination of the initial and confirmatory tests) currently used in this country. The research protocol to answer this question was registered with PROSPERO (no. CRD42016039710).

\section{Laboratory tests for HCV}

Laboratory tests for HCV infection can be divided into 3 categories based on what they detect: 1) antibodies to $\mathrm{HCV}$, 2) antibodies to HCV and HCV core antigen (i.e., antigenantibody tests) and 3) HCV RNA. ${ }^{16}$ Antibody tests include the third-generation enzyme-linked immunosorbent assay (ELISA), chemiluminescent immunoassay (CLIA), electrochemiluminescent immunoassay (ECLIA), chemiluminescent microparticle immunoassay (CMIA) and microparticle enzyme immunoassay (MEIA). Each commercial antibody testing kit uses slightly different sets of $\mathrm{HCV}$ antigens to bind and detect host antibodies (Table 1). Antigen-antibody tests include fourth-generation ELISAs; because they detect antibodies to $\mathrm{HCV}$, they have the same issues with false-positive results as third-generation ELISAs but higher sensitivity because they also detect $\mathrm{HCV}$ antigen. Molecular techniques that detect HCV RNA, such as polymerase chain reaction and nucleic acid amplification testing, are recommended as confirmatory tests, ${ }^{18}$ even though immunocompromised patients and those undergoing hemodialysis may have false-negative results. ${ }^{16}$ RNA-based testing can detect $\mathrm{HCV}$ before antibodies are produced (early infection) and can differentiate between current and resolved infection (i.e., fewer false-positive results). There is no perfect reference standard for $\mathrm{HCV}$ infection, and the US Centers for Disease Control and Prevention have recommended a testing strategy in which the initial test is an antibody test and the confirmatory test may be either RNA detection or serological methods similar to the index test. ${ }^{18}$

\section{Environmental scan of laboratory testing for HCV}

To determine the scope of our systematic review, we first performed an environmental scan of laboratory testing for $\mathrm{HCV}$ in Canada. Given that provincial and territorial laboratories generally perform most $\mathrm{HCV}$ testing, ${ }^{19}$ one author (G.C.) searched the grey literature on the websites of all provincial and territorial laboratories and the Canadian AIDS Treatment Information Exchange, and contacted the laboratories by email in February 2016, on behalf of the Canadian Task Force for Preventive Health Care, to obtain information about the tests and testing sequences used to diagnose $\mathrm{HCV}$ infection in Canada (Appendix 1, available at www. cmajopen.ca/content/4/4/E737/suppl/DC1). In most of the

\begin{tabular}{|c|c|c|}
\hline Test & $\begin{array}{l}\text { Examples of assays } \\
\text { (manufacturer) }\end{array}$ & $\begin{array}{l}\text { Antigens } \\
\text { (region of the genome) }\end{array}$ \\
\hline $\begin{array}{l}\text { Third-generation enzyme- } \\
\text { linked immunosorbent assay } \\
\text { (ELISA) }\end{array}$ & $\begin{array}{l}\text { HCV version } 3.0 \text { ELISA test system } \\
\text { (Ortho Clinical Diagnostics) }\end{array}$ & $\begin{array}{l}\text { c100-3 (NS3-NS4) } \\
\text { c33-c (NS3) } \\
\text { c22-3 (core) } \\
\text { NS5 }\end{array}$ \\
\hline $\begin{array}{l}\text { Chemiluminescent } \\
\text { immunoassay }\end{array}$ & $\begin{array}{l}\text { Architect } i 4000 \text { anti-HCV assay } \\
\text { (Abbott) } \\
\text { Vitros ECl anti-HCV assay (Ortho } \\
\text { Clinical Diagnostics) } \\
\text { Advia Centaur (Siemens) }\end{array}$ & $\begin{array}{l}\text { c22-3 (core) } \\
\text { c200 (NS3-NS4) } \\
\text { NS5 }\end{array}$ \\
\hline $\begin{array}{l}\text { Electrochemiluminescent } \\
\text { immunoassay }\end{array}$ & $\begin{array}{l}\text { Elecsys anti-HCV assay (Roche } \\
\text { Diagnostics) }\end{array}$ & $\begin{array}{l}\text { Core } \\
\text { NS3 } \\
\text { NS4 }\end{array}$ \\
\hline $\begin{array}{l}\text { Chemiluminescent } \\
\text { microparticle immunoassay }\end{array}$ & Architect anti-HCV (Abbott) & $\begin{array}{l}\mathrm{HCr} 43 \text { (core-NS3) } \\
\mathrm{c} 100-3 \text { (NS3-NS4) }\end{array}$ \\
\hline $\begin{array}{l}\text { Microparticle enzyme } \\
\text { immunoassay }\end{array}$ & AxSYM HCV version 3.0 (Abbott) & $\begin{array}{l}\text { HCr43 (fusion core eNS3) } \\
\text { c200 (NS3-NS4) } \\
\text { c100-3 (NS3-NS4) } \\
\text { NS5 }\end{array}$ \\
\hline
\end{tabular}


laboratories, the initial test used was an antibody test, such as the CLIA (British Columbia, Northwest Territory and Saskatchewan), CMIA (Alberta, Manitoba, New Brunswick, Newfoundland and Labrador, Nova Scotia and Ontario) or MEIA (Quebec). Confirmatory testing was typically performed by means of another immunoassay, such as the CMIA (British Columbia and Saskatchewan), CLIA (Ontario), fourth-generation ELISA (Alberta) or recombinant immunoblot assay (Manitoba and Quebec) rather than the polymerase chain reaction (New Brunswick, Newfoundland and Labrador, and Nova Scotia). Use of a second immunoassay to confirm HCV infection, ${ }^{20}$ rather than RNA detection, is also aligned with current US Centers for Disease Control and Prevention recommendations. ${ }^{18}$

\section{Literature search strategy}

We developed the literature search strategy with the help of a librarian at the University of Toronto Gerstein Science Information Centre (Appendix 2, available at www.cmajopen.ca/ content/4/4/E737/suppl/DC1). We systematically searched Ovid MEDLINE (1946-2016), Ovid MEDLINE In-Process and Other Non-Indexed Citations, and Ovid Embase using both controlled vocabulary and keywords. Because the oldest immunoassay of interest (ELISA version 3.0) was first marketed in $1993,{ }^{21}$ retrieval was limited to articles published in English or French between Jan. 1, 1990 and May 6, 2016. We excluded conference abstracts from the search results.

\section{Selection criteria}

Original research studies, systematic reviews and meta-analyses were eligible for inclusion. At least $80 \%$ of the study population had to be asymptomatic, nonpregnant, treatment-naïve adults with unknown liver enzyme values and unknown HCV status (e.g., general population, blood donors). We excluded studies involving high-risk groups such as patients undergoing hemodialysis, transplant/transfusion recipients, intravenous drug users and patients coinfected with other blood-borne infections, as well as blood bank specimens that had previously tested negative for $\mathrm{HCV}$ and specimen panels. The index test had to be the CLIA, ECLIA, CMIA, MEIA or ELISA version 3.0; rapid tests, tests performed on specimens other than blood (e.g., saliva), and sero- or genotyping tests were excluded. Within a given study, the reference test had to be different from the index test. In the absence of a perfect reference standard, 2 different sets of reference tests were eligible for inclusion: 1) inferior serological reference tests (CMIA, CLIA, ECLIA, MEIA, third- and fourth-generation ELISA or recombinant immunoblot assay), commonly used as confirmatory tests in Canada, and 2) superior RNA-based reference tests (polymerase chain reaction or nucleic acid amplification testing). The reference test had to be applied to some participants with a positive index test result as well as some of those with a negative index test result, so that a $2 \times 2$ table could be filled and sensitivity and/or specificity estimated. The setting had to resemble primary care (e.g., blood donation centre, population-based screening); hospital-based specialty clinics and inpatient hospital settings were excluded.

\section{Selection method}

Using DistillerSR software, 2 reviewers (G.C. and J.C.) independently screened all titles and abstracts using the predetermined selection criteria. Potentially relevant articles were retrieved, and the reviewers screened all full-text articles using the same selection criteria. Disagreements were resolved through discussion.

\section{Data extraction}

One reviewer (G.C.) extracted data on study characteristics and findings from each included study into Tables 2-4, as appropriate. Raw data were extracted to create $2 \times 2$ tables of index test(s) compared with reference test(s) for each study. The second reviewer (J.C.) verified the accuracy and completeness of the data extraction. Disagreements were resolved through discussion. We obtained additional data not reported in the published article from the authors of 1 included study. ${ }^{23}$

\section{Risk of bias assessment}

One reviewer (G.C.), who has previous experience conducting validation studies ${ }^{33,34}$ and performing statistical adjustment for verification bias, ${ }^{35}$ assessed the risk of bias and applicability of each included study using the Quality Assessment of Diagnostic Accuracy Studies version 2 (QUADAS-2) tool. QUADAS-2 focuses on 4 domains: patient selection, index test, reference test, and patient flow and timing of testing. ${ }^{36}$ The second reviewer (J.C.) verified the accuracy and completeness of the assessment. Disagreements were resolved through discussion.

\section{Statistical analysis}

We estimated sensitivity, specificity, positive predictive value, negative predictive value, false-positive rate, false-negative rate and $95 \%$ confidence intervals (CIs) using the raw data $(2 \times 2$ tables) extracted from the included studies. For studies that suffered from verification bias as a result of the sampling strategy used, we performed statistical adjustment of sensitivity and specificity estimates (and therefore of the false-positive and false-negative rates). ${ }^{31}$ Given the small number of included studies and the heterogeneity of index test-reference test pairings between studies, quantitative synthesis was not considered appropriate.

\section{Assessment of quality of body of evidence using GRADE methodology}

Whereas studies using inferior serological reference tests reflect current laboratory practice in several provinces and territories, they likely underestimate the true number of falsepositive results. For this reason, we further restricted the body of evidence to studies in which the index test was an antibody test (reflecting current laboratory practice) and the reference test detected RNA. Two reviewers (G.C. and J.C.) independently used GRADE (Grading of Recommendations Assessment, Development and Evaluation) methodology for diagnostic testing accuracy studies ${ }^{32,37-39}$ to assess the body of evidence; disagreements between reviewers were resolved through discussion. The GRADE criteria evaluate the evi- 


\section{Table 2: Characteristics of included studies}

\begin{tabular}{|c|c|c|c|c|c|c|c|}
\hline Author, year & $\begin{array}{c}\text { Country } \\
\text { (HCV } \\
\text { prevalence* })\end{array}$ & $\begin{array}{l}\text { Setting and study } \\
\text { period }\end{array}$ & $\begin{array}{l}\text { Funding source and } \\
\text { conflict(s) of interests }\end{array}$ & Study population & Study flow and timing & Index test & $\begin{array}{l}\text { Reference } \\
\text { test(s) }\end{array}$ \\
\hline $\begin{array}{l}\text { Denoyel et } \\
\text { al., }{ }^{15} 2004\end{array}$ & $\begin{array}{l}\text { France } \\
\text { (low) }\end{array}$ & NR & NR & $\begin{array}{l}5228 \text { participants: } 5015 \\
\text { random blood donors } \\
\text { and } 213 \text { inpatients }\end{array}$ & $\begin{array}{l}\text { Not specified; based on } \\
\text { results, all samples } \\
\text { underwent both index and } \\
\text { reference tests }\end{array}$ & $\begin{array}{l}\text { CLIA (Advia } \\
\text { Centaur HCV } \\
\text { assay) }\end{array}$ & $\begin{array}{l}\text { MEIA (AxSYM } \\
\text { HCV version } \\
3.0 \text { assay, } \\
\text { Abbott) }\end{array}$ \\
\hline $\begin{array}{l}\text { Tashkandy } \\
\text { et al., }{ }^{22} 2007\end{array}$ & $\begin{array}{l}\text { Saudi Arabia } \\
\text { (high) }\end{array}$ & $\begin{array}{l}\text { Immunology and } \\
\text { Serology } \\
\text { Department, } \\
\text { Al-Noor } \\
\text { Specialist } \\
\text { Hospital, } \\
\text { Makkah; study } \\
\text { period NR }\end{array}$ & NR & $\begin{array}{l}106 \text { male blood donors; } \\
\text { samples from patients } \\
\text { with diabetes or other } \\
\text { endocrine diseases and } \\
\text { autoimmune diseases } \\
\text { excluded }\end{array}$ & $\begin{array}{l}\text { All samples were aliquoted } \\
\text { into } 2 \text { portions, } 1 \text { for PCR, } \\
\text { the other for serological } \\
\text { testing }\end{array}$ & $\begin{array}{l}\text { ELISA version } \\
3.0 \text { (Murex } \\
\text { anti-HCV, } \\
\text { Abbott) }\end{array}$ & $\begin{array}{l}\text { LIA (Inno-LIA } \\
\text { HCV Ab III } \\
\text { Update, } \\
\text { Innogenetics) } \\
\text { RT-PCR (high } \\
\text { pure viral } \\
\text { nucleic acid } \\
\text { reagent set, } \\
\text { Roche) }\end{array}$ \\
\hline $\begin{array}{l}\text { Benouda et } \\
\text { al., }{ }^{23} 2009\end{array}$ & $\begin{array}{l}\text { Morocco } \\
\text { (high) }\end{array}$ & $\begin{array}{l}\text { Workplace blood } \\
\text { specimen } \\
\text { collection; } \\
\text { December } 2005 \\
\text { to April } 2007\end{array}$ & NR & $\begin{array}{l}8326 \text { adults from general } \\
\text { population with unknown } \\
\text { HCV serology }\end{array}$ & $\begin{array}{l}\text { Subset of } 158 / 161 \\
\text { ELISA-positive and } \\
\text { 100/8165 ELISA-negative } \\
\text { adults recalled to undergo } \\
\text { reference standard test } \\
\text { (verification bias); } 3 / 161 \\
\text { lost to follow-up; interval } \\
\text { between tests not specified }\end{array}$ & $\begin{array}{l}\text { ELISA version } \\
3.0 \text { (Murex } \\
\text { anti-HCV, } \\
\text { Abbott) }\end{array}$ & $\begin{array}{l}\text { MEIA (AxSYM } \\
\text { HCV version } \\
3.0 \text { assay, } \\
\text { Abbott) } \\
\text { RT-PCR } \\
\text { (Amplicor HCV } \\
\text { version 2.0, } \\
\text { Roche) }\end{array}$ \\
\hline $\begin{array}{l}\text { Rao et al., }{ }^{24} \\
2009\end{array}$ & $\begin{array}{l}\text { China } \\
\text { (high) }\end{array}$ & $\begin{array}{l}\text { Beijing Red } \\
\text { Cross Blood } \\
\text { Center and } \\
\text { Peking University } \\
\text { Hepatology } \\
\text { Institute; study } \\
\text { period NR }\end{array}$ & $\begin{array}{l}\text { Grants from Chinese } \\
\text { Basic Research } \\
\text { Foundation, National } \\
\text { Science and } \\
\text { Technology Key } \\
\text { Project, and Key } \\
\text { Clinical Research } \\
\text { Program of Ministry of } \\
\text { Health; conflict(s) of } \\
\text { interest NR, but } 1 \\
\text { author affiliated with } \\
\text { Aldatis }\end{array}$ & $\begin{array}{l}2559 \text { participants: } 2082 \\
\text { blood donors and } 477 \\
\text { patients including those } \\
\text { with various HCV } \\
\text { genotypes and non-C } \\
\text { hepatitis, pregnant } \\
\text { women and lipidemia } \\
\text { serum samples }\end{array}$ & $\begin{array}{l}\text { All samples underwent } \\
\text { both index and reference } \\
\text { tests "side-by-side" }\end{array}$ & $\begin{array}{l}\text { ELISA version } \\
4.0 \text { (EIAgen, } \\
\text { Adaltis) }\end{array}$ & $\begin{array}{l}\text { ELISA version } \\
3.0 \text { (HCV } \\
\text { version } 3.0 \\
\text { ELISA test } \\
\text { system, Ortho) }\end{array}$ \\
\hline $\begin{array}{l}\text { Ol et al., }{ }^{25} \\
2009\end{array}$ & $\begin{array}{l}\text { Cambodia } \\
\text { (moderate) }\end{array}$ & $\begin{array}{l}\text { Rural areas of } 2 \\
\text { Cambodian } \\
\text { provinces } \\
\text { (Battambang and } \\
\text { Pailin); May to } \\
\text { June } 2007\end{array}$ & $\begin{array}{l}\text { Sponsored by } \\
\text { European Plasma } \\
\text { Fraction Foundation } \\
\text { and Tromsø Mine } \\
\text { Victim Resource } \\
\text { Center, University } \\
\text { Hospital, North } \\
\text { Norway; conflict(s) of } \\
\text { interest NR }\end{array}$ & $\begin{array}{l}1200 \text { potential volunteer } \\
\text { blood donors: } 677 \\
\text { women, } 523 \text { men, mean } \\
\text { age } 32.8 \text { (range } 18-52 \text { ) } \\
\text { yr; sample stratified by } \\
\text { province ( } 600 \text { each) }\end{array}$ & $\begin{array}{l}\text { Subset of } 80 / 176 \\
\text { ELISA-positive and } \\
40 / 1024 \text { ELISA-negative } \\
\text { samples selected to } \\
\text { undergo reference test } \\
\text { (verification bias); all } \\
\text { samples underwent both } \\
\text { index and reference tests }\end{array}$ & $\begin{array}{l}\text { ELISA version } \\
4.0 \text { (Monolisa, } \\
\text { BioRad) }\end{array}$ & CMIA (Abbott) \\
\hline $\begin{array}{l}\text { Kosan et } \\
\text { al., }{ }^{26} 2010\end{array}$ & $\begin{array}{l}\text { Turkey } \\
\text { (high) }\end{array}$ & $\begin{array}{l}\text { Turkish Red } \\
\text { Crescent Çapa } \\
\text { Blood Centre of } \\
\text { Istanbul; } \\
\text { February } 2007 \text { to } \\
\text { March } 2008\end{array}$ & None declared & $\begin{array}{l}18200 \text { volunteer blood } \\
\text { donors: } 546 \text { women, } \\
17654 \text { men, mean age } \\
40 \text { (range 18-60) yr, } \\
18198 \text { were first-time } \\
\text { donors; participants } \\
\text { underwent mandatory } \\
\text { physical examination } \\
\text { before blood drawing }\end{array}$ & $\begin{array}{l}2 \text { sets of blood samples } \\
\text { collected from each } \\
\text { participant: } 1 \text { underwent } \\
\text { serological testing and the } \\
\text { other, NAT testing }\end{array}$ & $\begin{array}{l}\text { ELISA version } \\
3.0 \text { (Innotest } \\
\text { HCV Ab III, } \\
\text { Innogenetics) }\end{array}$ & $\begin{array}{l}\text { NAT (Procleix } \\
\text { Ultrio kit, } \\
\text { Chiron) }\end{array}$ \\
\hline $\begin{array}{l}\text { Park et al., }{ }^{27} \\
2012\end{array}$ & $\begin{array}{l}\text { South Korea } \\
\text { (low) }\end{array}$ & $\begin{array}{l}\text { "Routine HCV } \\
\text { screening"; } \\
\text { August } 2009 \text { to } \\
\text { January } 2011\end{array}$ & $\begin{array}{l}\text { Funding source not } \\
\text { reported, but Ortho } \\
\text { provided CLIA assay } \\
\text { kits; conflict(s) of } \\
\text { interest NR }\end{array}$ & $\begin{array}{l}1011 \text { serum samples from } \\
\text { participants undergoing } \\
\text { routine HCV screening }\end{array}$ & $\begin{array}{l}\text { Not specified; based on } \\
\text { results, all samples } \\
\text { underwent both index and } \\
\text { reference tests }\end{array}$ & $\begin{array}{l}\text { CLIA (Vitros } \\
\text { Anti-HCV } \\
\text { assay, Ortho) }\end{array}$ & $\begin{array}{l}\text { ECLIA } \\
\text { (Elecsys } \\
\text { anti-HCV test, } \\
\text { Roche) }\end{array}$ \\
\hline $\begin{array}{l}\text { Sommese et } \\
\text { al., }{ }^{28} 2014\end{array}$ & $\begin{array}{l}\text { Italy } \\
\text { (low) }\end{array}$ & $\begin{array}{l}\text { Second } \\
\text { University of } \\
\text { Naples; January } \\
\text { to June } 2013\end{array}$ & None declared & $\begin{array}{l}840 \text { volunteer blood } \\
\text { donors: } 275 \text { women, } 564 \\
\text { men, } \uparrow \text { mean age } 37.7 \text { yr }\end{array}$ & $\begin{array}{l}\text { All samples underwent } \\
\text { both index and reference } \\
\text { tests in parallel }\end{array}$ & $\begin{array}{l}\text { CMIA (Architect } \\
\text { i2000SR, } \\
\text { Abbott) }\end{array}$ & $\begin{array}{l}\text { ECLIA (Cobas } \\
\text { e411, Roche) }\end{array}$ \\
\hline $\begin{array}{l}\text { Arora et al., }{ }^{29} \\
2016\end{array}$ & $\begin{array}{l}\text { India } \\
\text { (moderate) }\end{array}$ & $\begin{array}{l}\text { Blood bank; } \\
\text { January } 2013 \text { to } \\
\text { March } 2014\end{array}$ & None declared & 21115 blood donors & $\begin{array}{l}\text { All samples underwent } \\
\text { both index and reference } \\
\text { tests in parallel }\end{array}$ & $\begin{array}{l}\text { ELISA version } \\
4.0 \text { (Monolisa } \\
\text { HCV Ag-Ab } \\
\text { Ultra, BioRad) }\end{array}$ & $\begin{array}{l}\text { NAT (Procleix } \\
\text { Ultrio kit, } \\
\text { Chiron) }\end{array}$ \\
\hline \multicolumn{8}{|c|}{$\begin{array}{l}\text { Note: } \mathrm{CLIA}=\text { chemiluminescent immunoassay, } \mathrm{CMIA}=\text { chemiluminescent microparticle immunoassay, ECLIA, electrochemiluminescent immunoassay, ELISA = enzyme- } \\
\text { linked immunosorbent assay, } \mathrm{HCV}=\text { hepatitis } \mathrm{C} \text { virus, } \mathrm{LIA}=\text { line immunoassay, MEIA = microparticle enzyme immunoassay, NAT = nucleic acid amplification test, NR = not } \\
\text { reported, PCR }=\text { polymerase chain reaction, } \mathrm{RT}-\mathrm{PCR}=\text { reverse-transcription polymerase chain reaction. } \\
\text { *Low }=<1.5 \% \text { moderate }=1.5 \%-3.5 \% \text {, high }=>3.5 \% .{ }^{30} \\
\dagger \text { As reported by the authors. }\end{array}$} \\
\hline
\end{tabular}




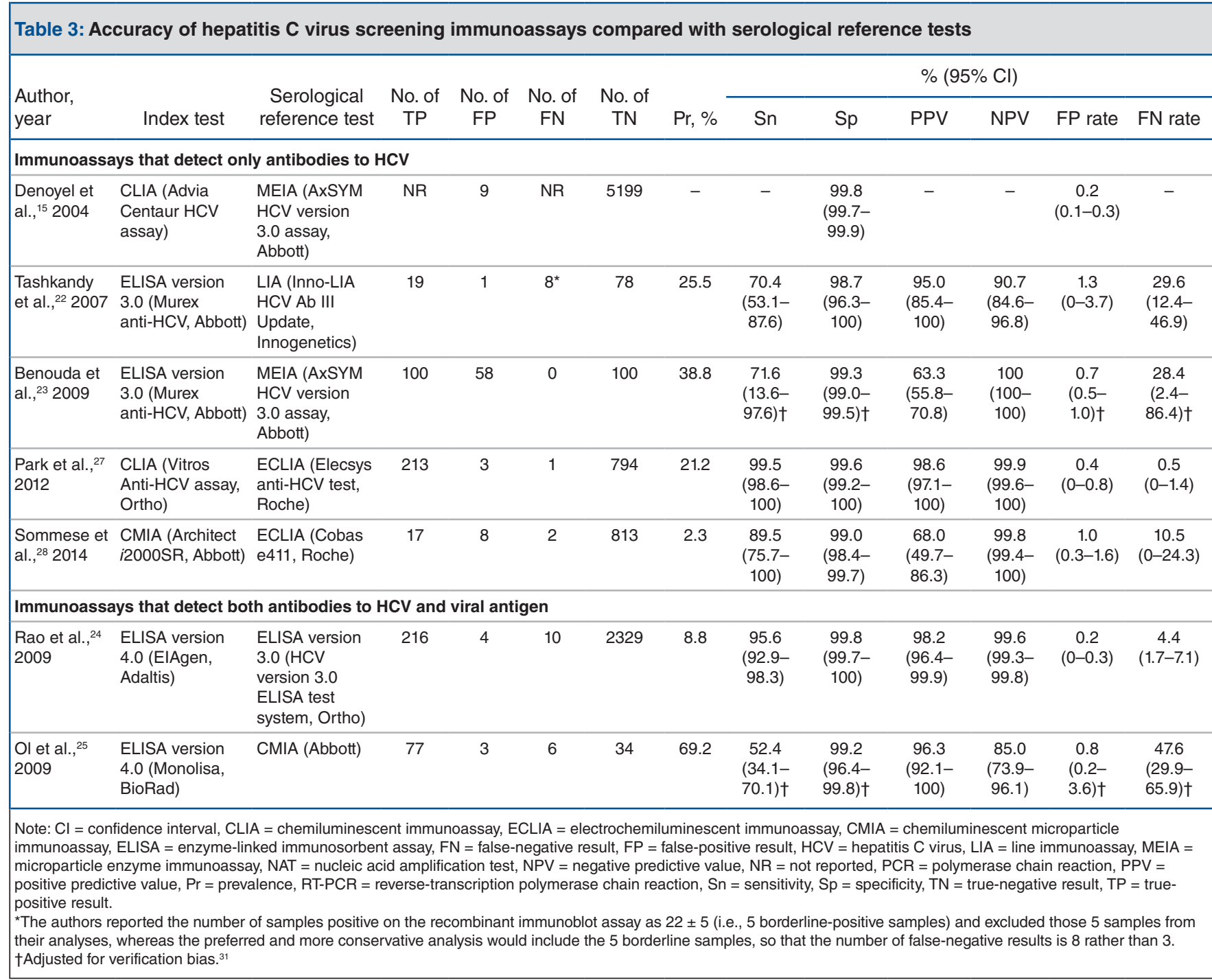

dence in terms of study design, risk of bias, indirectness, imprecision and publication bias. ${ }^{39}$

\section{Results}

\section{Literature search results and characteristics of included studies}

We identified 1537 studies, of which 81 underwent full-text review; 9 studies were included in our systematic review (Figure 1). A list of the excluded studies and each study's reason for exclusion is available in Appendix 3 (available at www.cmajopen. ca/content/4/X/E737/suppl/DC1). We did not identify any eligible studies that evaluated the 2 -step HCV screening procedure. Included studies (Table 2) were conducted in countries with low, ${ }^{15,27,28}$ moderate ${ }^{25,29}$ and high ${ }^{22-24,26} \mathrm{HCV}$ prevalence. ${ }^{30}$

\section{Accuracy of immunoassays compared with other immunoassays}

The sample size of studies comparing 2 immunoassays ranged from 106 to 5208 (Table 3). For antibody tests compared with other antibody tests, sensitivity ranged from $70.4 \%$ to $99.5 \%$ and specificity from $98.7 \%$ to $99.8 \%$. For an antigen-antibody test compared with an antibody test, sensitivity ranged from $52.4 \%$ to $95.6 \%$, and specificity was over $99 \%$.

\section{Accuracy of immunoassays compared with RNA detection}

The sample size of studies comparing an antibody test to RNA detection ranged from 106 to 21115 (Table 4). For antibody tests compared with RNA detection, sensitivity ranged from $61.0 \%$ to $81.8 \%$ and specificity ranged from $97.5 \%$ to $99.7 \%$. As expected, the antigen-antibody test performed better against RNA detection than the antibody tests did; its sensitivity was $90.2 \%$ and its specificity was $99.8 \%$. Also as expected, the number of false-positive results was higher and the positive predictive value was lower when comparing antibody tests to RNA detection than to other antibody tests.

\section{Assessment of risk of bias with QUADAS-2}

With respect to patient selection, only 2 studies involved routine HCV screening of the general population; ${ }^{23,27}$ the other 7 studies involved blood donor screening ${ }^{15,22,24-26,28,29}$ (QUADAS-2 


\begin{tabular}{|c|c|c|c|c|c|c|c|c|c|c|c|c|c|}
\hline \multirow[b]{2}{*}{ Author, year } & \multirow[b]{2}{*}{ Index test } & \multirow{2}{*}{$\begin{array}{l}\text { RNA-based } \\
\text { reference test }\end{array}$} & \multirow{2}{*}{$\begin{array}{l}\text { No. of } \\
\text { TP }\end{array}$} & \multirow{2}{*}{$\begin{array}{l}\text { No. of } \\
\text { FP }\end{array}$} & \multirow{2}{*}{$\begin{array}{l}\text { No. of } \\
\text { FN }\end{array}$} & \multirow{2}{*}{$\begin{array}{l}\text { No. of } \\
\text { TN }\end{array}$} & \multirow[b]{2}{*}{$\operatorname{Pr}, \%$} & \multicolumn{6}{|c|}{$\%(95 \% \mathrm{Cl})$} \\
\hline & & & & & & & & Sn & Sp & PPV & NPV & FP rate & FN rate \\
\hline \multicolumn{14}{|c|}{ Immunoassays that detect only antibodies to HCV } \\
\hline $\begin{array}{l}\text { Tashkandy et } \\
\text { al., }{ }^{22} 2007\end{array}$ & $\begin{array}{l}\text { ELISA version } \\
3.0 \text { (Murex } \\
\text { anti-HCV, } \\
\text { Abbott) }\end{array}$ & $\begin{array}{l}\text { RT-PCR (high } \\
\text { pure viral nucleic } \\
\text { acid reagent set, } \\
\text { Roche) }\end{array}$ & 18 & 2 & 7 & 79 & 23.6 & $\begin{array}{c}72.0 \\
(54.4- \\
89.6)\end{array}$ & $\begin{array}{c}97.5 \\
(94.2- \\
100)\end{array}$ & $\begin{array}{c}90.0 \\
(76.9- \\
100)\end{array}$ & $\begin{array}{c}91.9 \\
(86.1- \\
97.6)\end{array}$ & $\begin{array}{c}2.5 \\
(0-5.8)\end{array}$ & $\begin{array}{c}28.0 \\
(10.4- \\
45.6)\end{array}$ \\
\hline $\begin{array}{l}\text { Benouda et } \\
\text { al., }{ }^{23} 2009^{\star}\end{array}$ & $\begin{array}{l}\text { ELISA version } \\
3.0 \text { (Murex } \\
\text { anti-HCV, } \\
\text { Abbott) }\end{array}$ & $\begin{array}{l}\text { RT-PCR } \\
\text { (Amplicor HCV } \\
\text { version 2.0, } \\
\text { Roche) }\end{array}$ & 62 & 96 & 0 & 100 & 24.0 & $\begin{array}{c}61.0 \\
(8.9- \\
96.2) \dagger\end{array}$ & $\begin{array}{c}98.8 \\
(98.5- \\
99.0) \dagger\end{array}$ & $\begin{array}{c}39.2 \\
(31.6- \\
46.9)\end{array}$ & $\begin{array}{c}100 \\
(100- \\
100)\end{array}$ & $\begin{array}{c}1.2 \\
(1.0-1.5) \dagger\end{array}$ & $\begin{array}{c}39.0 \\
(3.8- \\
91.1) \dagger\end{array}$ \\
\hline $\begin{array}{l}\text { Kosan et al., }{ }^{26} \\
2010\end{array}$ & $\begin{array}{l}\text { ELISA version } \\
\text { 3.0 (Innotest } \\
\text { HCV Ab III, } \\
\text { Innogenetics) }\end{array}$ & $\begin{array}{l}\text { NAT (Procleix } \\
\text { Ultrio kit, Chiron) }\end{array}$ & 9 & 56 & 2 & 17784 & 0.1 & $\begin{array}{c}81.8 \\
(59.0- \\
100)\end{array}$ & $\begin{array}{c}99.7 \\
(99.6- \\
99.8)\end{array}$ & $\begin{array}{c}13.8 \\
(5.4- \\
22.2)\end{array}$ & $\begin{array}{c}100 \\
(100- \\
100)\end{array}$ & $\begin{array}{c}0.3 \\
(0.2-0.4)\end{array}$ & $\begin{array}{c}18.2 \\
(0-41.0)\end{array}$ \\
\hline \multicolumn{14}{|c|}{ Immunoassay that detects both antibodies to HCV and viral antigen } \\
\hline $\begin{array}{l}\text { Arora et al., }{ }^{29} \\
2016\end{array}$ & $\begin{array}{l}\text { ELISA version } \\
4.0 \text { (Monolisa } \\
\text { Ag-Ab Ultra, } \\
\text { BioRad) }\end{array}$ & $\begin{array}{l}\text { NAT (Procleix } \\
\text { Ultrio kit, Chiron) }\end{array}$ & 37 & 46 & 4 & 21028 & 0.2 & $\begin{array}{c}90.2 \\
(81.2- \\
99.3)\end{array}$ & $\begin{array}{c}99.8 \\
(99.7- \\
99.8)\end{array}$ & $\begin{array}{c}44.6 \\
(33.9- \\
55.3)\end{array}$ & $\begin{array}{c}100 \\
(100- \\
100)\end{array}$ & $\begin{array}{c}0.2 \\
(0.2-0.3)\end{array}$ & $\begin{array}{c}9.8 \\
(0.7-18.8)\end{array}$ \\
\hline \multicolumn{14}{|c|}{$\begin{array}{l}\text { Note: } \mathrm{Cl}=\text { confidence interval, } \mathrm{ELISA}=\text { enzyme-linked immunosorbent assay, } \mathrm{FN}=\text { false-negative result, } \mathrm{FP}=\text { false-positive result, } \mathrm{HCV}=\text { hepatitis } \mathrm{C} \text { virus, } \mathrm{NAT}=\text { nucleic } \\
\text { acid amplification test, } \mathrm{NPV}=\text { negative predictive value, } \mathrm{PPV}=\text { positive predictive value, } \mathrm{Pr}=\text { prevalence, } \mathrm{RT}-\mathrm{PCR}=\text { reverse-transcription polymerase chain reaction, } \mathrm{Sn}= \\
\text { sensitivity, } \mathrm{Sp}=\text { specificity, } \mathrm{TN}=\text { true-negative result, } \mathrm{TP}=\text { true-positive result. } \\
{ }^{*} \text { The authors did not report on the comparison of ELISA version } 3.0 \text { to } \mathrm{PCR} \text {; these data were obtained through personal communication (Amina Benouda, Hôpital universitaire } \\
\text { Cheikh-Zaid, Rabat, Morocco: personal communication, 2016). } \\
\text { TAdjusted for verification bias }{ }^{32}\end{array}$} \\
\hline
\end{tabular}

assessment, Appendix 4, available at www.cmajopen.ca/content/ 4/X/E737/suppl/DC1). The 9 included studies reported on 11 different index test-reference test pairings: 5 studies compared antibody tests to other antibody tests, ${ }^{15,22,23,27,28} 2$ studies compared antigen-antibody tests to antibody tests, ${ }^{24,25} 3$ studies compared antibody tests to RNA detection ${ }^{22,23,26}$ (Amina Benouda, Hôpital universitaire Cheikh-Zaid, Rabat, Morocco: personal communication, 2016), and 1 study compared an antigen-antibody test to RNA detection. ${ }^{29}$ With respect to patient flow and timing of testing, 7 studies applied the index and reference tests to all samples in parallel. ${ }^{15,22,24,26-29}$ Two studies had verification bias as a result of applying the reference test to a larger proportion of samples that had tested positive on the index test relative to those that had tested negative on the index test. . $^{23,25}$

\section{Assessment of quality of body of evidence with GRADE methodology}

We considered 3 studies comparing antibody tests to RNA detection for inclusion in the body of evidence. ${ }^{22,23,26}$ One of the 3 studies $^{23}$ was excluded because, as a result of there being no false-negative results, the correction of the sensitivity estimate for verification bias was overly conservative ${ }^{40}$ and the uncorrected sensitivity was too biased to be meaningful. Another study $^{22}$ was excluded because, based on its results, the study prevalence of $\mathrm{HCV}$ was very high, at $23.6 \%$; this suggested that either the study population was not reflective of the general population or a case-control design was used (in either case, it did not meet our inclusion criteria). The quality of evidence in the remaining study ${ }^{26}$ was assessed as very low (Table 5); this study reported a sensitivity of $81.8 \%$ (95\% CI 59.0\%-100\%) and a specificity of $99.7 \%$ (95\% CI 99.6\%-99.8\%). Assuming an HCV seroprevalence rate of $0.96 \%$, as in the general Canadian population, ${ }^{2}$ instead of the rate of $0.1 \%$ among the 17840 blood donors in the study, ${ }^{38}$ the positive predictive value would be $72.7 \%$ (95\% CI $66.2 \%-78.8 \%$ ), and the negative predictive value would be $99.8 \%$ (95\% CI 99.8\%-99.9\%). Applying this study's findings to 1000 people drawn from the general Canadian population, we would expect 8 (95\% CI 6-10) truepositive results, 987 (95\% CI 986-988) true-negative results, 3 (95\% CI 2-4) false-positive results and 2 (95\% CI 0-4) falsenegative results (Table 5).

\section{Interpretation}

In our systematic review of the evidence on the accuracy of antibody tests compared with other immunoassays and RNA detection for screening asymptomatic adults for HCV infection, we found that the sensitivity of antibody tests was highly variable $(52.4 \%-99.5 \%)$ and the specificity was high $(97.5 \%-$ $99.8 \%)$. The lack of a perfect reference test for HCV infection raises concerns that these estimates are biased. In particular, when an inferior serological reference test that shares the same risk of false-positive results as the index test is used, the specificity could be overestimated. As expected, we found that there were more false-positive results when comparing antibody tests to superior RNA-based reference tests than to inferior serological reference tests. Bias correction of the specificity estimate could make the difference even greater. This finding highlights a potential problem with using an inferior 


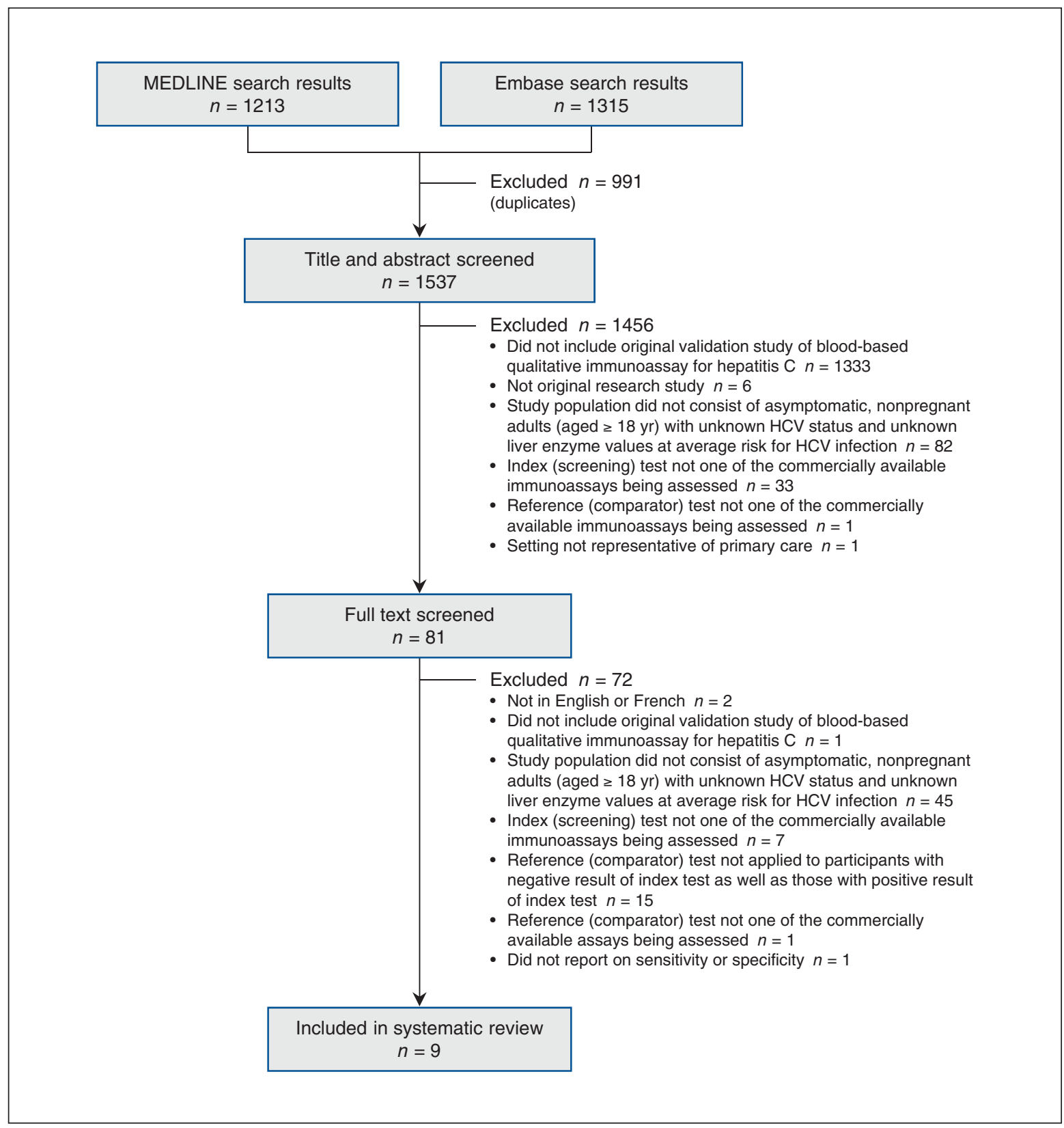

Figure 1: Flow diagram of study selection. $\mathrm{HCV}=$ hepatitis $\mathrm{C}$ virus.

serological reference test as a confirmatory test $\mathrm{t}^{18,20}$ when screening the general population for HCV infection. Our assessment of the body of evidence using GRADE methodology led us to focus on a single "least-biased" study, ${ }^{26}$ in which the sensitivity of the ELISA version 3.0 compared with nucleic acid amplification testing was $81.8 \%$ (95\% CI 59.0\%$100 \%$ ) and the specificity $99.7 \%$ (95\% CI $99.6 \%-99.8 \%$ ). Assuming an $\mathrm{HCV}$ seroprevalence rate of $0.96 \%$, as in the general Canadian population, ${ }^{2}$ the positive predictive value would be $72.7 \%$ (95\% CI $66.2 \%-78.8 \%$ ); in other words, 1 of every 4 positive ELISA version 3.0 test results would be a false-positive result.

The only other systematic review of the accuracy of $\mathrm{HCV}$ antibody testing was performed in the context of the US
Preventive Services Task Force's 2004 recommendation on HCV screening. ${ }^{8}$ However, the populations of the studies included in that review (i.e., patients undergoing hemodialysis, patients with histologically verified hepatitis, patients admitted to hospital with suspected acute/chronic hepatitis, blood donors with persistently elevated liver enzyme values and blood donors who previously screened positive for $\mathrm{HCV}$ ) did not reflect the general population; therefore, those findings are not directly comparable to ours. ${ }^{21}$ When the US Preventive Services Task Force revised its recommendation on HCV screening in $2013,{ }^{3}$ it did not reassess the accuracy of HCV screening tests, despite the introduction of new immunoassays (i.e., CLIA, ECLIA, CMIA, MEIA) since its 2004 systematic review. Similarly, when the 
Table 5: GRADE (Grading of Recommendations Assessment, Development and Evaluation) ${ }^{32,37-39}$ assessment of the quality of the body of evidence regarding the accuracy of antibody tests compared to RNA detection in screening for chronic hepatitis $\mathrm{C}$ virus infection in 1 cross-sectional study (cohort-type accuracy study)

\begin{tabular}{|c|c|c|c|c|c|c|c|c|c|c|}
\hline \multirow[b]{2}{*}{ Outcome } & \multirow{2}{*}{$\begin{array}{l}\text { Author, yr; } \\
\text { no. of } \\
\text { participants }\end{array}$} & \multicolumn{5}{|c|}{ Quality assessment domain* } & \multicolumn{3}{|c|}{$\begin{array}{l}\text { Pretest probability, \%†; expected no. } \\
\text { per } 1000 \text { patients tested } \\
(95 \% \mathrm{Cl})\end{array}$} & \multirow[b]{2}{*}{$\begin{array}{l}\text { Overall } \\
\text { quality } \ddagger\end{array}$} \\
\hline & & $\begin{array}{l}\text { Risk of } \\
\text { bias }\end{array}$ & Indirectness & Inconsistency & Imprecision & $\begin{array}{l}\text { Publication } \\
\text { bias }\end{array}$ & 0.96 & 0.61 & 1.34 & \\
\hline $\begin{array}{l}\text { True } \\
\text { positive }\end{array}$ & \multirow{2}{*}{$\begin{array}{l}\text { Kosan et } \\
\text { al., }{ }^{26} 2010 \\
11\end{array}$} & \multirow[t]{2}{*}{$++++\S$} & \multirow[t]{2}{*}{$++\emptyset$} & \multirow[t]{2}{*}{$+++^{\star \star}$} & \multirow[t]{2}{*}{$++† \dagger$} & \multirow[t]{2}{*}{ None } & $\begin{array}{c}8 \\
(6-10)\end{array}$ & $\begin{array}{c}5 \\
(4-6)\end{array}$ & $\begin{array}{c}11 \\
(8-13)\end{array}$ & Very low \\
\hline $\begin{array}{l}\text { False } \\
\text { negative }\end{array}$ & & & & & & & $\begin{array}{c}2 \\
(0-4)\end{array}$ & $\begin{array}{c}1 \\
(0-2)\end{array}$ & $\begin{array}{c}2 \\
(0-5)\end{array}$ & - \\
\hline $\begin{array}{l}\text { True } \\
\text { negative }\end{array}$ & \multirow{2}{*}{$\begin{array}{l}\text { Kosan et } \\
\text { al., }{ }^{26} 2010 \\
17840\end{array}$} & \multirow[t]{2}{*}{$++++\S$} & \multirow[t]{2}{*}{++9} & \multirow[t]{2}{*}{$+++^{\star \star}$} & \multirow[t]{2}{*}{ ++++†† } & \multirow[t]{2}{*}{ None } & $\begin{array}{c}987 \\
(986-988)\end{array}$ & $\begin{array}{c}991 \\
(990-992)\end{array}$ & $\begin{array}{c}984 \\
(983-985)\end{array}$ & Very low \\
\hline $\begin{array}{l}\text { False } \\
\text { positive }\end{array}$ & & & & & & & $\begin{array}{c}3 \\
(2-4)\end{array}$ & $\begin{array}{c}3 \\
(2-4)\end{array}$ & $\begin{array}{c}3 \\
(2-4)\end{array}$ & - \\
\hline \multicolumn{11}{|c|}{$\begin{array}{l}\text { Note: } \mathrm{Cl}=\text { confidence interval. } \\
{ }^{*} \text { The overall quality is determined by downgrading, from a rating of "high" ( } 4 \text { plus signs), for any concerns as follows: } 0 \text { (no concerns), }-1 \text { (serious concerns) or }-2 \text { (very } \\
\text { serious concerns). } \\
\text { †The values correspond to the HCV seroprevalence estimate in the general Canadian population of } 0.96 \%(95 \% \mathrm{Cl} 0.61 \%-1.34 \%){ }^{2} \\
\text { †The } 4 \text { possible ratings for overall quality are high, moderate, low and very low. We had serious or very serious concerns regarding several of the domains, resulting in an } \\
\text { overall rating of "very low." } \\
\left.\text { §Based on the Quality Assessment of Diagnostic Accuracy Studies version } 2 \text { tool, }{ }^{36} \text { the risk of bias was assessed to be low (Appendix } 4\right) \text {. } \\
\text { IThis domain was downgraded by } 2 \text { points because 1) the study population was different from that in our research question (Turkish volunteers who underwent a mandatory } \\
\text { physical examination before donating blood versus the general Canadian population) and } 2 \text { ) the study answered an indirect question about the accuracy of diagnostic } \\
\text { testing rather than directly assessing the effectiveness of screening the general population for HCV infection. } \\
\star \star \\
\star\end{array}$} \\
\hline
\end{tabular}

World Health Organization published its HCV screening guideline in $2014,{ }^{41}$ it did not reassess screening test accuracy, instead citing a 2001 report $^{42}$ on simple/rapid test accuracy and a 2001 report $^{43}$ on the accuracy of the ELISA version 3.0 or later immunoassay, both involving blood panels not reflective of the general population. Because antibody tests have not been adequately evaluated for population-based HCV screening, and because the availability of a safe, valid and reliable screening test is a fundamental consideration of any screening recommendation or program, ${ }^{10-12}$ this highlights a knowledge gap and brings into question the evidence basis for these recommendations.

\section{Limitations}

Our findings are limited by the paucity and low quality of the available evidence published in English and French. In particular, we were unable to locate any studies of the accuracy of the CLIA, CMIA or MEIA (the HCV screening tests most commonly used in Canada) compared to RNA detection for HCV screening in the general population. The applicability of our findings to the general Canadian population is limited because most included studies were conducted among blood donors, and people eligible to donate blood are at lower risk for bloodborne infections such as HCV infection than the general population. Rapid and point-of-care tests were beyond the scope of our review. Although these tests are important for reaching some vulnerable populations, most HCV testing in Canada is laboratory-based; $;^{19}$ in addition, a systematic review of the accuracy of rapid tests was recently published. ${ }^{44}$

\section{Conclusion}

The availability of a safe, valid and reliable screening test is a primordial consideration for decision-making about screening, but our study has shown that further research is needed to adequately characterize the accuracy of antibody tests used to screen the general population for chronic HCV infection. We focused on the accuracy of HCV screening tests; however, several other important factors must be considered when making decisions about HCV screening, including the benefits and harms of screening, the benefits and harms of treatment for screen-detected cases, the cost-effectiveness of screening and patient preferences related to screening. A review of the evidence related to these considerations is beyond the scope of the present study, but such a review is being performed by other investigators in the context of the Canadian Task Force on Preventive Health Care's upcoming guidelines on HCV screening. To help inform decision-making about HCV screening, we encourage jurisdictions that have already adopted populationbased (birth cohort) screening for chronic HCV infection to carefully evaluate and report on the accuracy of antibody tests as well as screening benefits and harms.

\section{References}

1. Hepatitis C in Canada: 2005-2010 surveillance report. Ottawa: Public Health Agency of Canada; 2012. Available: http://publications.gc.ca/collections/ collection_2012/aspc-phac/HP40-70-2012-eng.pdf (accessed 2016 Mar. 29).

2. Trubnikov M, Yan P, Archibald C. Estimated prevalence of hepatitis C virus infection in Canada, 2011. Can Commun Dis Rep 2014;40:429-36.

3. Chou R, Cottrell EB, Wasson N, et al. Screening for hepatitis C virus infection in adults: a systematic review for the U.S. Preventive Services Task Force. Ann Intern Med 2013;158:101-8. 
4. Provincial Infectious Diseases Advisory Committee on Communicable Diseases, Ontario Agency for Health Protection and Promotion (Public Health Ontario). Recommendations for the public health response to hepatitis $\mathrm{C}$ in Ontario. Toronto: Queen's Printer for Ontario; 2014. Available: www. publichealthontario.ca/en/eRepository/Recommendations_Public_Health_ Response_Hepatitis_C.pdf (accessed 2016 May 9).

5. Hepatitis C: essential information for professionals and guidance on testing. London (UK): National Health Service; 2004. Available: www.nhs.uk/hepatitisc/ SiteCollectionDocuments/pdf/essential-information-for-professionals-andguidance-on-testing.pdf (accessed 2016 Mar. 24).

6. Kohli A, Shaffer A, Sherman A, et al. Treatment of hepatitis C: a systematic review. 7AMA 2014;312:631-40.

7. Moyer VA; U.S. Preventive Services Task Force. Screening for hepatitis C virus infection in adults: U.S. Preventive Services Task Force recommendation statement. Ann Intern Med 2013;159:349-57.

8. Chou R, Clark EC, Helfand M; U.S. Preventive Services Task Force. Screening for hepatitis $C$ virus infection: a review of the evidence for the U.S. Preventive Services Task Force. Ann Intern Med 2004;140:465-79.

9. Recommendations on screening for hepatitis C. Ottawa: Canadian Task Force on Preventive Health Care; updated 2015 Nov. 19. Available: http://canadian taskforce.ca/ctfphc-guidelines/2015-hepatitis-c/guidelines-on-screening-for -hepatitis-c/ (accessed 2016 June 12).

10. Wilson JM, Jungner YG. Principles and practice of mass screening for disease [article in Spanish]. Bol Oficina Sanit Panam 1968;65:281-393.

11. Andermann A, Blancquaert I, Beauchamp S, et al. Revisiting Wilson and Jungner in the genomic age: a review of screening criteria over the past 40 years. Bull World Health Organ 2008;86:317-9.

12. Criteria for appraising the viability, effectiveness and appropriateness of a screening programme. London (UK): Public Health England; updated 2015 Oct. 23. Available: https://www.gov.uk/government/publications/evidence -review-criteria-national-screening-programmes/criteria-for-appraising-the -viability-effectiveness-and-appropriateness-of-a-screening-programme (accessed 2016 Apr. 27).

13. Giuberti T, Ferrari C, Marchelli S, et al. Long-term follow-up of antihepatitis $\mathrm{C}$ virus antibodies in patients with acute non $\mathrm{A}$ nonB hepatitis and different outcome of liver disease. Liver 1992;12:94-9.

14. Hajarizadeh B, Grebely J, Dore GJ. Epidemiology and natural history of HCV infection. Nat Rev Gastroenterol Hepatol 2013;10:553-62.

15. Denoyel G, van Helden J, Bauer R, et al. Performance of a new hepatitis C assay on the Bayer ADVIA Centaur Immunoassay System. Clin Lab 2004;50: $75-82$.

16. Saludes V, Gonzalez V, Planas R, et al. Tools for the diagnosis of hepatitis C virus infection and hepatic fibrosis staging. World 7 Gastroenterol 2014;20: 3431-42.

17. Villar LM, Cruz HM, Barbosa JR, et al. Update on hepatitis B and C virus diagnosis. World 7 Virol 2015;4:323-42.

18. Centers for Disease Control and Prevention. Testing for HCV infection: an update of guidance for clinicians and laboratorians. MMWR Morb Mortal Wkly Rep 2013;62:362-5

19. Hepatitis C: testing in your region. Toronto: Canadian AIDS Treatment Information Exchange; updated 2010.

20. Vermeersch P, Van Ranst M, Lagrou K. Validation of a strategy for HCV antibody testing with two enzyme immunoassays in a routine clinical laboratory. 7 Clin Virol 2008;42:394-8.

21. Colin C, Lanoir D, Touzet S, et al. Sensitivity and specificity of third-generation hepatitis $\mathrm{C}$ virus antibody detection assays: an analysis of the literature. $\mathcal{F}$ Viral Hepat 2001;8:87-95.

22. Tashkandy MA, Khodari YA, Ibrahim AM, et al. Evaluation of the available anti-HCV antibody detection tests and RT-PCR assay in the diagnosis of hepatitis C virus infection. Saudi 7 Kidney Dis Transpl 2007;18:523-31.

23. Benouda A, Boujdiya Z, Ahid S, et al. Prevalence of hepatitis $\mathrm{C}$ virus infection in Morocco and serological tests assessment of detection for the viremia prediction [article in French]. Pathol Biol (Paris) 2009;57:368-72.

24. Rao HY, Ren FR, Guan WL, et al. Evaluation of the performance of the EIAgen HCV test for detection of hepatitis C virus infection. 7 Virol Methods 2009; $162: 203-7$

25. Ol HS, Bjoerkvoll B, Sothy S, et al. Prevalence of hepatitis B and hepatitis C virus infections in potential blood donors in rural Cambodia. Southeast Asian 7 Trop Med Public Health 2009;40:963-71.

26. Kosan E, Kocazeybek B, Altunay H, et al. Can the nucleic acid amplification test (NAT) be an alternative to the serologic tests? A prospective study, the results of 18,200 blood donors from the Turkish Red Crescent. Transfus Apher Sci 2010;43:269-72.

27. Park Y, Seok Y, Choi J, et al. Performance evaluation of the Vitros antihepatitis $\mathrm{C}$ virus antibody assay for use in clinical laboratories. Clin Biochem 2012;45:175-7

28. Sommese L, Sabia C, Paolillo R, et al. Screening tests for hepatitis B virus, hepatitis $\mathrm{C}$ virus, and human immunodeficiency virus in blood donors: evalu- ation of two chemiluminescent immunoassay systems. Scand 7 Infect Dis 2014;46:660-4.

29. Arora S, Doda V. Role of signal-to-cut-off ratios of anti-hepatitis C virus antibody by enzyme immunoassays along with ID-NAT for screening of whole blood donors in India. Asian 7 Transfus Sci 2016;10:75-8.

30. Mohd Hanafiah K, Groeger J, Flaxman AD, et al. Global epidemiology of hepatitis $C$ virus infection: new estimates of age-specific antibody to HCV seroprevalence. Hepatology 2013;57:1333-42.

31. Irwig L, Glasziou PP, Berry G, et al. Efficient study designs to assess the accuracy of screening tests. Am F Epidemiol 1994;140:759-69.

32. Schünemann HJ, Oxman AD, Brozek J, et al.; GRADE Working Group. Grading quality of evidence and strength of recommendations for diagnostic tests and strategies [published erratum in BMF 2008;336:0-b]. BMF 2008;336: 1106-10.

33. Cadieux G, Tamblyn R. Accuracy of physician billing claims for identifying acute respiratory infections in primary care. Health Serv Res 2008;43:2223-38.

34. Cadieux G, Buckeridge DL, Jacques A, et al. Accuracy of syndrome definitions based on diagnoses in physician claims. BMC Public Health 2011;11:17-26.

35. Cadieux G, Tamblyn R, Buckeridge DL, et al. Validation of diagnostic groups based on health care utilization data should adjust for sampling strategy. Med Care 2015 Mar. 27 [Epub ahead of print]. DOI:10.1097/MLR. 0000000000000324

36. Whiting PF, Rutjes AW, Westwood ME, et al. QUADAS-2 Group. QUADAS-2: a revised tool for the quality assessment of diagnostic accuracy studies. Ann Intern Med 2011;155:529-36.

37. Brozek JL, Akl EA, Jaeschke R, et al.; GRADE Working Group. Grading quality of evidence and strength of recommendations in clinical practice guidelines: Part 2 of 3 . The GRADE approach to grading quality of evidence about diagnostic tests and strategies. Allergy 2009;64:1109-16.

38. Hsu J, Brożek JL, Terracciano L, et al. Application of GRADE: making evidencebased recommendations about diagnostic tests in clinical practice guidelines. Implement Sci 2011;6:62.

39. Gopalakrishna G, Mustafa RA, Davenport C, et al. Applying Grading of Recommendations Assessment, Development and Evaluation (GRADE) to diagnostic tests was challenging but doable. 7 Clin Epidemiol 2014;67:760-8.

40. Cronin AM, Vickers AJ. Statistical methods to correct for verification bias in diagnostic studies are inadequate when there are few false negatives: a simulation study. BMC Med Res Methodol 2008;8:75.

41. Guidelines for the screening, care and treatment of persons with hepatitis C infection. Geneva: World Health Organization; 2014. Available: www.who. int/hiv/pub/hepatitis/hepatitis-c-guidelines/en/ (accessed 2016 May 6).

42. Hepatitis C assays: operational characteristics (phase I): report 1. Geneva: World Health Organization; 2001. Available: http://apps.who.int/medicinedocs/en/d/ Js15208e/ (accessed 2016 Mar. 29).

43. Hepatitis C assays: operational characteristics (phase I): report 2. Geneva: World Health Organization; 2001. Available: http://apps.who.int/iris/ handle/10665/67061 (accessed 2016 Mar. 29).

44. Shivkumar S, Peeling R, Jafari Y, et al. Accuracy of rapid and point-of-care screening tests for hepatitis $\mathrm{C}$ : a systematic review and meta-analysis. Ann Intern Med 2012;157:558-66.

Affiliations: Dalla Lana School of Public Health (Cadieux, Campbell), University of Toronto, Toronto, Ont.; Department of Epidemiology, Biostatistics and Occupational Health (Dendukuri), McGill University, Montréal, Que.

Contributors: Geneviève Cadieux developed the protocol, performed the systematic review (first reviewer), performed the data analysis and drafted the manuscript. Jennifer Campbell helped develop the study protocol, performed the systematic review (second reviewer) and revised the manuscript critically for important intellectual content. Nandini Dendukuri helped develop the study protocol, oversaw the data analysis and revised the manuscript critically for important intellectual content. All of the authors approved the final version to be published and agreed to act as guarantors of the work.

Acknowledgements: The authors are grateful to Roland Grad, member of the working group on HCV screening of the Canadian Task Force for Preventive Health Care, for his helpful comments on the study design, findings and manuscript. The authors thank Barry Pakes, Public Health and Preventive Medicine residency program director at the University of Toronto, for his support and editorial suggestions.

Supplemental information: For reviewer comments and the original submission of this manuscript, please see www.cmajopen.ca/content/4/4/ E737/suppl/DC1 